\title{
Surgical Management of a Caustic Ingestion in a Gastric Bypass Patient: A Case Report
}

Van Boxstael E, Terwagne $\mathbf{N}$ and Deswysen $\mathbf{Y}^{*}$ Catholic University of Louvain, Upper GI Surgical Unit, Belgium

*Correspondling author: Deswysen Y, Catholic University of Louvain, Upper GI Surgical Unit, Upper GI Surgical Unit, Service de Chirurgie et Transplantation Abdominale, Cliniques universitaires Saint-Luc, 10, Avenue Hippocrate, B-1200 Bruxelles, Belgium

Received: March 04, 2021; Accepted: March 18, 2021 Published: March 25, 2021

\begin{abstract}
Introduction: Bariatric surgery is recognized as the most effective treatment for obesity. Increased rate of psychological disorders has been noted after surgery. However, no case of caustic ingestion after bariatric surgery and its surgical management has been reported in the literature.

Presentation of Case: A 48-year-old woman, who underwent a Rouxen-Y gastric bypass 9 years ago, ingested caustic substances as a suicide attempt, causing necrosis of the entire alimentary limb without severe lesion of esophageal tract or gastric pouch. During exploratory laparotomy, resection of the alimentary limb and gastrostomy in the neogastric pouch were performed. Three months later, the patient presented to the emergency room with a dislodged gastrostomy tube. Exploratory laparotomy was performed with restoration of anatomical continuity via gastro-gastric anastomosis.
\end{abstract}

Discussion: Bariatric surgery is correlated to a higher postoperative risk of psychological disorders until suicide attempt. This case is the first description of caustic ingestion after Roux-en-Y gastric bypass and its surgical management. It underlines the importance for bariatric teams to consider psychological aspect of surgical patients pre- and postoperatively.

Keywords: Caustic ingestion; Gastric bypass; Surgical management

\section{Abbreviations}

CT-scan: Computer-Tomography

\section{Introduction}

Bariatric surgery is considered as the most effective treatment for obese patients. The Roux-en-Y gastric bypass is still one of the most common procedure [1]. However, this surgery is related to an increased risk of postoperative psychological disorders, self-harm and suicides. After the intervention, studies show an approximately $50 \%$ higher risk of self-harm and suicide $[2,3]$.

Moreover, the initial attitude in case of caustic ingestion consists in the realisation of emergency laboratory tests, a CT-scan and an oesophagogastroduodenoscopy. Depending on the extent lesions, a conservative or surgical approach may be chosen [4].

We report an unique case of caustic ingestion as a suicide attempt of a 48-year-old woman who underwent Roux-en- $Y$ gastric bypass nine years ago and its surgical management.

\section{Case Presentation}

A 48-year-old woman, who underwent a Roux-en-Y gastric bypass in 2011, presented to the emergency room of another institution on October 2020 following the ingestion of caustic substances as a suicide attempt. Her medical history revealed severe depression. The type and the amount of substance ingested remained unknown. She suffered from abdominal pain and impaired general condition. She was referred to our expert hospital immediately transferred to the intensive care unit for support. Physical examination was normal except abdominal pain in the left upper quadrant.
Blood tests were remarkable for hyperleukocytosis and high inflammation. The abdominal CT-scan showed necrosis of almost all the alimentary limb, without any signs of gastrointestinal perforation. No esophageal or thoracic impairment was describe.

Upper gastrointestinal endoscopy revealed circumferential superficial ulcers of all the oesophagus (stage 2a regarding the Zargar classification) and of the gastric pouch and necrosis of the alimentary limb.

An emergency exploratory laparotomy was performed with resection of the alimentary limb. The jejuno-jejunostomy anastomosis was preserve because without no lesion. As the oesophagus and neogastric pouch seemed macroscopically viable, a Malecot gastrostomy tube was placed in the neogastric pouch. No additional lesion was detected. Finally, alimentary jejunostomy was performed. No postoperative complication occurred. Feeding was carried out exclusively through the feeding jejunostomy. Psychiatric follow-up was organized by the liaison psychiatry team before the discharge.

Three months later, the patient presented to the emergency room with a dislodged gastrostomy tube. Few abdominal complaints were noted. Physical examination and blood sample were fast normal. Because of the risk of peritonitis, an exploratory laparotomy in emergency was performed. The intraoperative oesophagogastroscopy showed a normal aspect of both the oesophageal and neogastric mucosa. No stenosis or inflammatory change were noted. Therefore, anatomical continuity was restored with a manual gastrogastric termino-terminal anastomosis, between the neogastric pouch and the excluded portion of stomach. 
Refeeding was gradually achieved and the postoperative course was uneventful. The postoperative follow-up at 1 month was unremarkable. Psychiatric follow-up was continued.

\section{Discussion}

Bariatric surgery has become increasingly common due to the worldwide obesity epidemic. The goal of a successful treatment is to achieve long-term weight loss and a remission or improvement of related comorbidities.

Although bariatric surgery is the most effective therapy for weight loss, it is now well described that patients undergoing this procedure have a higher prevalence of postoperative mental disorders. Some authors described a higher rate of suicide and self-harm after bariatric surgery around approximately 50 percent $[1,2]$. Obviously, obese patients with a history of psychiatric disorder are at higher risk of decompensation [3].

Caustic ingestion is a frequent emergency and can have serious consequences. Next to children who accidentally ingest household products, adults with suicidal intents constitute the second most atrisk age group for caustic ingestion.

In case of a severe burn of upper-gastrointestinal tract necrosis could occur, even to perforation. After several weeks, cicatrisation mechanisms can lead to stenosis. Initial emergency management aims to focus on patient survival, treat early complications and avoid chronic sequelae, while making sure the nutritional status stays correct. Gastrointestinal injuries should be evaluated by using emergency laboratory tests, oesophagogastroscopy (Zargar classification) and CT-scan. Emergency surgery is the gold standard when the initial assessment suggests transmural necrosis of the gastrointestinal tract. Furthermore, a psychiatric approach and psychological support are essential [5].

We described the first case in literature of caustic ingestion after Roux-en-Y gastric bypass, with necrosis of the alimentary limb without any severe esophageal or gastric damage.

For our patient, the initial assessment consisted of emergency blood tests, CT-scan and endoscopic evaluation as recommended. Laparotomy was performed because of the intestinal necrosis in the context of gastric bypass.

In this case, three months after surgery, the gastrostomy tube was accidentally removed. The need for a surgical intervention allowed the possibility to restore anatomical continuity, providing that peroperatory oesophago-gastroscopy showed a normal oesophageal and gastric tract. As this the case, so gastric bypass reversal could be performed. A hand-sewn anastomosis between the neogastric pouch and the excluded stomach was performed in the same way as a reverse bypass carried out for severe denutrition or severe dumping syndrome [6]. Side effect of this type of procedure can occurred consisting of weight regain, severe gastroesophageal reflux disease, persistent abdominal pain, but also sepsis, anastomotic leak, haemorrhage, reoperation and readmission [7]. None of these complications have been described for our patient.

This highlights the importance of a good follow-up after procedure, including screening for self-harm behaviour and suicide risk assessment. For our patient, a follow-up with the psychologist of the multidisciplinary team was set up after the operation.

\section{Conclusion}

Considering the increased risk of self-harm and suicide after bariatric surgery, this unique case of caustic ingestion after Roux-en-Y gastric bypass illustrates the absolute necessity of multidisciplinary evaluation including the psychological aspect before considering bariatric surgery. It highlights also the importance of a postoperative psychological follow-up, especially in patients with psychiatric history.

\section{References}

1. Buchwald H, Oien DM. Metabolic/bariatric surgery worldwide 2011. Obesity surgery. 2013; 23: 427-436.

2. Adams TD, Gress RE, Smith SC, Halverson RC, Simper SC, Rosamond WD, et al. Long-term mortality after gastric bypass surgery. The New England journal of medicine. 2007; 357: 753-761.

3. Bhatti JA, Nathens AB, Thiruchelvam D, Grantcharov T, Goldstein BI, Redelmeier DA. Self-harm Emergencies after Bariatric Surgery: A PopulationBased Cohort Study. JAMA surgery. 2016; 151: 226-232.

4. Fieux F, Villa A, Losser M, Cattan P. Ingestion de produits caustiques chez l'adulte. Reanimation. 2009; 18: 606-616.

5. Shoar S, Nguyen T, Ona MA, Reddy M, Anand S, Alkuwari MJ, et al. Rouxen-Y gastric bypass reversal: a systematic review. Surgery for obesity and related diseases: official journal of the American Society for Bariatric Surgery. 2016; 12: 1366-1372

6. Pernar LI, Kim JJ, Shikora SA. Gastric bypass reversal: a 7-year experience. Surgery for obesity and related diseases: official journal of the American Society for Bariatric Surgery. 2016; 12: 1492-1498.

7. Chirica M, Bonavina L, Kelly MD, Sarfati E, Cattan P. Caustic ingestion Lancet (London, England). 2017; 389: 2041-2052. 